\title{
Influence of the Emergence of Gait Recognition System on Human Society
}

\author{
Xin chen ${ }^{1}$ Tianqi yang ${ }^{2}$ \\ ${ }^{1}$ jinan university
}

\begin{abstract}
As an emerging biometric identification technology, gait recognition is getting more and more attention from researchers. By researching the gait characteristics mainly when the target is walking, it realizes the recognition of target identity. As the unique feature in longdistance recognition of human body, gait will not be affected by the facial form, makeup, and cloth of human body, and has become the hot topic of research in current intelligent identification. With a large amount of investment by research institutions in various countries, gait recognition is rapidly developing to marketization. The emergence of gait recognition, which is as intellectualized as to the current common recognition system of biometric features, is an irresistible trend. In the paper, the basic principle of gait recognition is mainly introduced; the main difficulties encountered in the researches at home and abroad are analyzed; the solutions are proposed; and the application prospect of gait recognition and its main influences on various major disciplines are analyzed.
\end{abstract}

Keywords: gait recognition, development, influence, prospect

\section{Introduction}

From the point of information science, biometric features recognition is an issue of traditional pattern recognition, which identifies the human. The biometric fea- tures of everyone have different uniqueness and different unchangeable stability in a period from others, which are difficult to forge and counterfeit, thus it is safe, reliable and accurate to recognize identity by biometric identification technology. However, no biometric feature is perfect. The first generation of biometric identification technologies, including widely-used fingerprint recognition, face recognition, iris recognition and hand geometry recognition, mostly needs the cooperation of the target monitored, and even needs the target to complete necessary action before realization. These methods are cumbersome, slow, inconvenient, and not accepted easily [1] [2] [3]. With the continuous development of current makeup and face-lifting technologies, the proportion of human's five features can be changed through different makeup (Fig. 1). Meanwhile, in that the criminal means are increasingly intelligent and technological, and that the disguise and makeup technologies of criminals who mostly are mastered, wear gloves or take other measures in crime are higher and higher, the important information like fingerprint cannot be collected. The first generation of identity recognition technology will face challenges of anti-counterfeiting and antitheft.

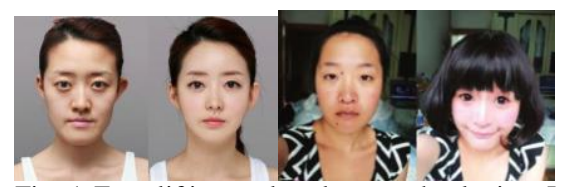

Fig. 1 Face-lifting and makeup technologies, Left: before and after face-lifting; Right: before and after makeup 
Gait recognition is a new recognition technology of biometric features, which aims at realizing the recognition of personal identity or the detection of physiological, pathological and psychological features according to people's ways of working. As a biometric recognition technology, gait recognition has the advantages that other biometric recognition technologies do not have, which are recognition potential from long distance or in low video quality, and difficulty in hiding or disguising. The infrared gait recognition technology can play a greater role especially in the night. The obvious advantages of gait recognition technology include: firstly, gait recognition needs neither cooperation of the target monitored nor touch with the recognition device; secondly, gait recognition can recognize the identity of the pedestrian from long distance, even monitor the identity of the pedestrian in wider range (500 feet or longer); thirdly, the gait features are coordinated actions of the parts of body, which are difficult to change for some time, unless serious disease or injury, and cannot be counterfeited and imitated [4] [5]. It can be seen that gait recognition combines several technologies including computer vision, pattern recognition, and video/image sequence processing with broad application prospect. Therefore, it has aroused great interests of many researchers at home and abroad, and become the forefront direction focused by the field of biomedical information detection in recent years.

As the extraction of gait features is getting more and more accurate currently, the extraction algorithm is increasingly simple and convenient. The emergence and wide application of gait recognition system will become an inexorable trend, thus the intelligent monitoring level in the world will rise to a new height, and bring new subject and challenge in the fields of public security, public regulation, medicine, and psychology, etc.

\section{Principle and Method of Gait Recognition System}

The key technologies involved in remote identity recognition system based on gait recognition include video processing, image processing, and pattern recognition [6]. The recognition process is shown in Fig. 2.

\subsection{Extraction Methods of Gait Fea- tures}

The extraction method based on feature does not need to construct model, but to propose assumption on the object to be researched, and extract feature parameter from the gait by using various statistical values of space-time pattern produced in moving target in image sequence. Hereinto, the most commonly used one is the contour feature of gait. This kind of algorithm has no direct relationship with the body structure and dynamic feature of walk, but it is sensitive to the changes of background and light signal. The recognition capability will be greatly influenced once shelter appears in the scene.

As for gait recognition, it is crucial to solve the problem of shelter. However, the gait analysis based on model has the advantage on this aspect. The reason is that the model is established on the basis of move mode of human in sequence image. It can not only reflect the current change, but also estimate the changes in the past and the future. The gait recognition method based on model prebuilds model, and obtains the model parameter through matching of model and twodimensional image sequence.

The two methods above have their own advantages and disadvantages. Gait feature is transforming from multidimension to one-dimension and from surface-to-point to simplification. 


\subsection{Extraction Methods of Gait Fea- tures}

Gait recognition is mainly divided into three processes including pretreatment in earlier state, feature extraction and model matching. The main focus of current research lies in the extraction of gait feature, which is to describe the basic features of human's walking in the form of data vector. To complete the main work to complete recognition process during model matching, there are two main methods currently:

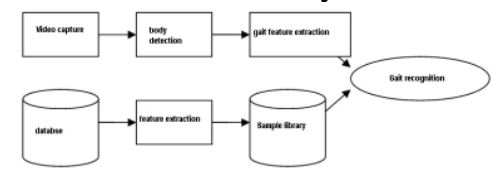

Fig. 2 Schematic Diagram of Gait Recognition System

Template matching method is widely used in the field of pattern recognition, and is a basic feature matching. It converts image sequence into a group of static shape mode, and compare with the behavior specimen prestored in the process of recognition. Taking the similarity as the standard of classification, the $\mathrm{K}$ Nearest Neighbor is usually used for recognition. The merit of template matching technology is low in computation complexity, and simple to realize, while it is sensitive to the changes of noise and movement time interval.

Template matching is actually a method based on distance measurement. When using the method in gait recognition, the connection relationship among postures implied in gait sequence is ignored. The distance between image and sample posture produces observation vector of low dimension, hereby to train the parameter of statistic model for gait recognition. Statistic model mainly consists of Neural Network method, Support Vector Machine (SVM) method, and Hidden Markov Model (HMM) method.

\section{Technological Difficulties and Solu- tions}

\subsection{Technological Difficulties}

The profile of pedestrian is easily affected by clothes and accompanying articles. With the development of clothing industry, people's pursuit of fashion clothes is increasingly intense, while lots of clothes would cover the legs of pedestrian, so that the legs which can best reflect gait features are covered. However, as remote intelligent monitoring system, gait recognition system cannot make three-dimensional measurement including weighting and direct touch, but can only obtain the features through twodimensional pictures, thus it brings difficulties to the extraction of gait features. In addition, on different ground, the legs and feet of people will bring about irregular changes. Thereby certain interference is caused to gait features.

Under slant view angle, the profile of pedestrian shot and fluctuation will change greatly, because the irregularity of human body leads to different profile features on different sides, thus difficulties are caused to the extraction of gait features.

As the gait features extracted are generally floating points, and the current statistical method on the operational accuracy of floating points is not optimistic, the result can be obtained through rounding off operation. In the following work, the recognizer aimed specially at vector operation of gait feature can be worked out for high-accuracy and high-speed recognition.

\subsection{Solutions}

The problems above can be solved through abstract expression of body shape. The gait recognition is transformed from profile change into fluctuation change through abstraction, thus the gait recognition will not be influenced by changeful 
body profile. In a word, gait recognition is an edge discipline involving many disciplines including physiology, neuroscience, and psychology, etc. Therefore, the research results of biophysiological model, behavioral model, and mental model must be used for reference in the future to obtain the maximum of various information and integrate organically to use, so as to improve the correct recognition rate of gait recognition system.

\section{Development Prospect and Expec- tation}

\subsection{Influence on Sociology}

At present, every country pays high attention to such an issue about how to conduct all-weather, automatic and real-time remote monitoring and identity recognition in important security department and sensitive places of the country. Identity recognition technology based on gait features is, however, one of the effective means to solve the problem [7]. Gait recognition machine can be installed at the entrances and exits of airports and other important places of various countries, forming a huge intangible antiterrorist net to enhance safety degree of human society and built a harmonious and safe habitat for humanity. Fig. 3 shows pedestrians' pictures shot by road camera. As there are only back profile pictures, all the features like traditional face, fingerprint and iris lose their effects at the moment. However, gait recognition system can identify the identity of pedestrian through a video of such a pedestrian who is walking currently.

The emergence of gait recognition system in various monitored places will make the criminals who are good at execute crime through masking and making up find no place to hide. As gait has the characteristics of long distance and difficulty in disguising, gait recognition does not need to shoot the front profile of human body as face recognition does, but only a background can trace all the information [8]. Besides, it can release all the gait features extracted to the recognition systems in various street for realtime monitoring. It will give an alarm when meeting pedestrian with similar gait features, thus it will greatly reduce the crime rate in the world, and the monitoring capability will be promoted to a new height.

\subsection{Influence on Kinematics}

Gait recognition problem is a subproblem of kinematic analysis of human, hence the research results of gait recognition may be extended to solve other problems in the field of computer vision, for example distinguishing different movement (movements like walking, running and hitting the ball in playing tennis), and interpretation of sign language [10], etc. By comparing of features in sports, it can provide professional and accurate guidance for the performance of athletes, so as to make new records for several sport events.

\subsection{Influence on Medicine}

Gait assessment is also an important aspect in daily clinical practice of neurology, rheumatology, orthopedics, and rehabilitation medicine. Patients who suffer from diseases of nervous system or musculoskeletal system which influence their walking ability need gait analysis to assess whether the patients have abnormal gait and assess the nature and degree of abnormal gait, so as to provide necessary basis for analyzing the causes of abnormal gait, correcting abnormal gait, and making therapeutic schedule. The basic process and mechanism of abnormal gait can be understood through gait analysis. Thereby the force, joint angle and muscular activity can be analyzed in detail, and then related medical research can be carried out [9]. 
The emergence of gait recognition also brings some controversies. As gait recognition system can identify the identity of pedestrian through a video of back profile, the monitor capability in public places is greatly improved. The application of it in monitoring places makes people feel that their privacy has been infringed, and disenables them to obtain their private space by making up, while it is noticeable to disguise the gait. Many stars often prevent them from being recognized by wearing masks and sunglasses in public places, but the system can identify their real identities instantly through a video that they walk, which has great impact on the travel of public figures. The mature gait recognition system has not appeared in the world by now. The British scientists put forward to get rid of the influence of clothing changes on gait recognition through clothing cucoloris, so as to improve the identification accuracy. As this method infringes the personal privacy even more, it will inevitably bring larger controversy if using on a large scale. Therefore, once gait recognition system appears, it is bound to be used under certain supervision measures. As to the degree of supervision and the acceptance of people, it will be a new research direction for ethics.

\section{Conclusion}

The development and application of gait recognition technology can not only help developing related products through which great economic benefits will be obtained, but also promote the development of related disciplines, including image processing, pattern recognition, optics, electronics, physiology and computer application, and bring huge social benefits; it will provide a new idea for researches of medicine and kinematics, which makes people understand their physiological and pathological character- istics through their gait features. However, it may be restricted by corresponding ethics and morals for its excessive recognition capability at the same time of bringing benefits.

\section{References}

[1] Dong Xu, Yi Huang. Human Gait Recognition Using Patch Distribution Feature and LocalityConstrained Group Sparse Representation[J].IEEE Transactions on Image Processing. 21(1), 316326(2012)

[2] Fan Zhang, Xinhong Zhang, Kui Cao. Contour extraction of gait recognition based on improved GVF Snake model[J]. Computers and Electrical Engineering.38(4),882-890(2012).

[3] David Cunado, Mark S. Nixon. Automatic extraction and description of human gait models for recognition purposes[J]. Computer Vision and Image Understanding.90(1),1-41 (2003).

[4] Derawi Mohammad Omar, Ali Hazem, Cheikh Faouzi Alaya. Gait recognition using time-of-flight sensor[C]. Lecture Notes in Informatics (LNI), Proceedings-Series of the Gesellschaft fur Informatik (GI). Gesellschaft fur Informatik (GI), Ahrstrabe 45, 53175 Bonn, Germany. pp, 187 194(2011)

[5] Tao Yu, Jianhua Zou. Research on Gait Recognition Based on the Combination of HMM and Bayes Rules[J]. Chinese Journal of Computers. 35(2), 386-396(2012). (In Chinese)

[6] Zhang Peng, Xiaojuan Wu, Jun Yang. Multiview Gait Recognition Algorithm Based on the Parameters of the Limb Length[J].Journal of Automation.33(2), 211-213(2007). (In Chinese)

[7] Yanmei Chai,Tian Xia, Wenying Han,Haifeng Li. Progress in the study of gait recognition. [J]. computer science.39(6),10-15(2012).

[8] Zhaojun Xue,Jingna Qi. The status and progress of researches on gait recognition [J].Journal of biomedical engineering.25(5),1217-1221(2008).

[9] Qiong Cheng,Bingsong Zhou,Bo Fu. Design of gait recognition system[J]. Journal of Hubei University of Technology.23(5),12-14(2008).

[10] Vogler C, Sun H, Met axas D. A framework for motion recogn- ition w ith applications to American sign language and gait recogn- ition[ A] . In: Proceedings of Workshop on Human Motion [ C ] ,Aust in T exas, USA, 33 -38(2000). 